\title{
Barriers and attitudes reported by Canadian emergency physicians regarding the initiation of buprenorphine/naloxone in the emergency department for patients with opioid use disorder
}

\author{
Tyson Savage $^{1} \cdot$ Marshall Ross $^{1}$
}

Received: 1 May 2021 / Accepted: 5 August 2021 / Published online: 25 September 2021

() The Author(s), under exclusive licence to Canadian Association of Emergency Physicians (CAEP)/ Association Canadienne de Médecine d'Urgence (ACMU) 2021, corrected publication 2021

\begin{abstract}
Objectives The primary objective of this study is to identify emergency physician reported barriers to initiating patients on buprenorphine/naloxone in the emergency department (ED) for treatment of opioid use disorder. Secondary objectives include (1) physician reported attitudes about initiating buprenorphine/naloxone in the ED, and (2) comparison of barriers reported based on urban versus rural practice setting.

Methods An online survey was distributed to a convenience sample of attending emergency physicians and resident physicians using the Canadian Association of Emergency Physicians (CAEP) research survey email distribution network.

Results The survey was sent to 1299 email accounts registered with the CAEP research survey network. We received 121 responses, which is a response rate of 9.3\%. The completion rate was 118/121 (97.5\%). Most respondents 113/118 (95.7\%) reported at least one barrier that prevents them from initiating buprenorphine/naloxone in the ED. The top three reported barriers were (1) lack of allied health care staff who were trained to assist in starting patients on buprenorphine/naloxone in the ED and to help arrange follow-up, (2) time constraints related to patient education on the appropriate and safe use of buprenorphine/naloxone, and (3) access to follow-up resources. The majority of respondents agreed buprenorphine/naloxone was an evidence-based treatment for opioid use disorder and that it is important to make changes in their ED to better facilitate this practice. There was no statistically significant difference in the number of physicians reporting each barrier based on urban versus rural practice setting.

Conclusions In this convenience sample of physicians working in urban and rural Canadian emergency departments, most physicians perceive barriers that inhibit their ability to initiate buprenorphine/naloxone for patients with opioid use disorder, but overall there is support for making changes to better facilitate this practice.
\end{abstract}

Keywords Opioiud use disorder $\cdot$ Buprenorphine $\cdot$ Addictions

\section{Résumé}

Objectifs L'objectif principal de cette étude est d'identifier les obstacles signalés par les médecins urgentistes à la mise sous buprénorphine/naloxone des patients dans le service des urgences pour le traitement du trouble de l'usage des opiacés. Les objectifs secondaires comprennent (1) les attitudes déclarées par les médecins concernant l'administration de buprénorphine/ naloxone aux urgences, et (2) la comparaison des obstacles déclarés en fonction du milieu de pratique urbain par rapport au milieu rural.

Méthodes Un sondage en ligne a été distribué à un échantillon de commodité d'urgentistes titulaires et de médecins résidents en utilisant le réseau de distribution de courriels de recherche de l'Association canadienne des médecins d'urgence (ACMU).

Tyson Savage

tyson.savage@ucalgary.ca

1 Department of Emergency Medicine, University of Calgary, 2nd Floor Foothills Medical Centre, 1403 29th Street, NW,

Calgary AB T2N 2T9, Canada 
Résultats L'enquête a été envoyée à 1299 comptes de messagerie enregistrés auprès du réseau d'enquête de recherche ACMU. Nous avons reçu 121 réponses, soit un taux de réponse de 9,3\%. Le taux d'achèvement était de 118/121 (97,5\%). La plupart des répondants 113/118 (95,7\%) ont signalé au moins un obstacle qui les empêche d'initier la buprénorphine/ naloxone aux urgences. Les trois principaux obstacles signalés sont les suivants : (1) le manque de personnel paramédical formé pour aider à mettre les patients sous buprénorphine/naloxone aux urgences et à organiser le suivi (2) les contraintes de temps liées à l'éducation des patients sur l'utilisation appropriée et sûre de la buprénorphine/naloxone, et (3) l'accès aux ressources de suivi. La majorité des répondants ont convenu que la buprénorphine/naloxone était un traitement fondé sur des données probantes pour les troubles liés à l'utilisation d'opiö̈des et qu'il est important d'apporter des changements à leur service d'urgence pour mieux faciliter cette pratique. Il n'y avait pas de différence statistiquement significative dans le nombre de médecins signalant chaque obstacle selon que le milieu de pratique était urbain ou rural.

Conclusions Dans cet échantillon de commodité de médecins travaillant dans des services d'urgence canadiens urbains et ruraux, la plupart des médecins perçoivent des obstacles qui les empêchent d'initier la buprénorphine/naloxone pour les patients souffrant de troubles liés à l'utilisation d'opiö̈des, mais dans l'ensemble, ils sont favorables à des changements pour mieux faciliter cette pratique.

\section{Clinician's capsule}

\section{What is known about the topic?}

Starting patients on buprenorphine/naloxone for opioid use disorder is an evidence-based practice but is not widely adopted in Canadian EDs.

\section{What did this study ask?}

What are the main barriers and attitudes reported by Canadian emergency Physicians regarding starting patients on buprenorphine/naloxone in the ED?

\section{What did this study find?}

Most physicians reported at least one barrier that prevents starting buprenorphine/naloxone in the ED, despite positive attitudes towards the practice.

\section{Why does this study matter to clinicians?}

The barriers documented in this study identify areas to target to enhance adherence to this guideline-recommended practice in Canadian EDs

\section{Introduction}

Opioid use disorder is a major contributor of morbidity and mortality to Canadians and increasing evidence suggests the emergency department (ED) can play a meaningful role in treatment through the initiation of buprenorphine/naloxone [1-4]. Randomized control trial data show patients initiated on buprenorphine/naloxone in the ED were more likely to be engaged in addiction treatment, have less days of selfreported illicit opioid use, and use less inpatient addiction resources in 30-day follow-up [1]. An observational study demonstrated an associated decrease in all-cause mortality in patients who received buprenorphine in the 12 months following a non-fatal overdose [2]. The Canadian Medical Association guidelines for management of opioid use disorders provide a strong recommendation for buprenorphine/ naloxone as a first-line treatment, and in a position statement, the Canadian Association of Emergency Physicians (CAEP) recommends initiating buprenorphine/naloxone for patients with opioid use disorder in the $\operatorname{ED~}[3,4]$.

Despite the initiation of buprenorphine/naloxone in the ED being an evidence-based and guideline-recommended practice, a 2020 survey of Canadian emergency physicians indicates that it is not frequently prescribed [5]. Barriers reported from physicians working in urban centres in the United States included lack of local protocols, time constraints, and arranging appropriate follow-up [6-8]. In a qualitative study that interviewed 32 Canadian emergency physicians, also from mostly urban centres, perceived barriers included lack of experience, lack of ED resources, and lack of opioid agonist therapy training [9]. We seek to further describe and document the perceived barriers and attitudes of Canadian emergency physicians working in both urban and rural centres to better understand why adherence to guidelines is low.

Our primary objective is to identify the number of Canadian emergency physicians who report proposed barriers as substantial enough that it prevents them from initiating buprenorphine/naloxone in the ED. Secondary objectives include to: (1) report emergency physician attitudes about buprenorphine/naloxone, (2) report recommended resources, and (3) compare rates of each proposed barrier based on urban versus rural practice setting.

\section{Methods}

\section{Survey design, content, and distribution}

We created a list of proposed barriers and attitude statements based on studies reporting perceived barriers and attitudes of emergency physicians working in urban centres in the US, as well as with consultation with members of 
the Alberta Health Services Strategic Clinical Network who were involved with a multi-site quality improvement project regarding ED buprenorphine/naloxone initiation [6-8]. No formal survey method was used.

Survey participants were asked to respond 'yes' or 'no' to the list of proposed barriers based on whether or not it was a barrier that prevented them from starting buprenorphine/naloxone in the ED. Participants were asked to read the attitude statements and respond on a 5-point Likert scale (Strongly Disagree, Disagree, Neutral, Agree, Strongly Agree). Additional barriers and recommended resources were reported as optional free-text responses. Free text responses were grouped into themes by two research members with consensus. Demographic data collected included number of years in practice, prior buprenorphine/naloxone training, and main practice setting.

A 21-question survey was created using Qualtrics (https:// www.qualtrics.com) online survey software. The full survey is included in the online supplementary material. Potential risks and benefits were outlined, and informed consent was implied by survey completion. No personal identifiers were collected, and survey software was password protected. The survey was piloted to four physicians for feedback on language, clarity, and functionality, for which minor edits to functionality were made.

The target population was Canadian emergency physicians and residents planning to practice in the ED. Convenience sampling was performed using an established research survey distribution network managed by CAEP. The survey invitation was emailed to CAEP physician members on November 23, 2020 and the last response received was on December 29, 2020. The invitation was sent once without gift incentive. All mandatory questions needed to be answered for the response to be considered complete and used in analysis.

\section{Outcome measures}

The primary outcome is the number of respondents responding 'yes' to each proposed barrier as preventing them from initiating buprenorphine/naloxone in the ED.

Secondary outcomes include: (1) other proposed barriers (2) reported attitudes on buprenorphine/naloxone initiation in the ED, (3) proposed resources, and (4) comparison of barriers reported based on urban versus rural practice setting.

\section{Data analysis}

Data analysis was performed using IBM SPSS Statistics (Ver. 27). Percentage of respondents citing each proposed barrier, recommended resource, and attitude statement was calculated based on total number of respondents.
The Fischer's Exact test was used to assess for a statistically significant difference $(p<0.05)$ between number of respondents replying 'yes' to each proposed barrier based on practice setting.

\section{Research ethics}

The research protocol for this study was approved by the University of Calgary Research Ethics Board (Ethics ID: REB19-2026).

\section{Results}

The survey was emailed to 1299 attending and resident physicians with a response rate of 121/1299 (9.3\%) and a completion rate of 118/121 (97.5\%). Full demographic information can be found in the online supplemental materials. Most respondents were attending physicians (83.9\%). Responses came from 9 different provinces and 1 territory with 58.5\% from academic hospital, $21.2 \%$ from rural hospital, and 20.3\% from urban community hospital practice settings.

Our primary outcome is reported in Table 1 along with other barriers reported as optional free-text responses. Most respondents (95.7\%) agreed to at least one proposed barrier or reported an optional barrier.

Secondary outcomes including suggested resources and physician attitudes are reported in Table 1 and Fig. 1, respectively. The comparison of barriers reported based on urban versus rural practice setting can be found in the online supplemental figure. There was no statistically significant difference between the number of respondents responding 'yes' to each proposed barrier based on urban versus rural practice setting.

\section{Discussion}

\section{Interpretation of findings}

The finding that $95.7 \%$ of physicians surveyed reported at least one barrier preventing them from initiating buprenorphine/naloxone in the ED is a signal that there is still a need to better facilitate this guideline-recommended practice in Canadian EDs. The top two most frequently reported barriers relate to the perceived time constraints and need for more support from allied health care staff in the process of initiation and arranging follow-up. The most common optional response for other barriers was "length of time required for patient to enter severe enough withdrawal in ED". We interpret these findings to represent an issue at two levels. First, individual physicians may not have the education, experience, or institutional support with buprenorphine/naloxone 
Table 1 Survey responses to proposed barriers and optional responses for other perceived barriers and suggested resources

Is this a barrier that prevents you from initiating patients with buprenorphine/ Number of respondents replying 'Yes' $(\%) N=118$ naloxone in the ED? (mandatory 'yes' or 'no' response)

Lack of allied health care staff trained to assist in starting buprenorphine/naloxone and arranging follow-up

Time constraints relating to educating patients on the appropriate and safe use

Access to follow-up resources

Lack of timely access to buprenorphine/naloxone in the ED

Being unsure of which patients would benefit

Lack of familiarity with the risks

Lack of familiarity with the benefits

$29(24.6)$

What other barriers prevent you from initiating patients with buprenorphine/ naloxone in the ED? (optional free-text response)

Length of time required to wait for patient to enter severe enough withdrawal to start buprenorphine in ED

Lack of training/experience with buprenorphine

Patient not wanting to start/restart on buprenorphine

Department culture (perceived lack of support from leadership or that no one else in department is prescribing buprenorphine/naloxone)

Local licensing restrictions on prescribing buprenorphine

Feeling unsure if a patient has been abstinent long enough to start buprenorphine/ concerns about precipitated withdrawal

Patient leaves before addictions consultant or ED MD with buprenorphine license can discuss/initiate

No micro-dosing ${ }^{\text {a }}$ protocol

Need for screening tool for OUD in ED

Need for pre-printed discharge instructions

Desire for clear, step-by-step guideline

Patient under police arrest affecting follow-up

What resources would aid emergency physicians in initiating buprenorphine/ naloxone more regularly? (optional free-text response)

Allied health care staff (RN, SW, addiction's resource worker) trained to assist in induction/arranging follow-up

Availability of "Rapid Access Addictions" Clinics

Increased access to educational resources (free online access resources, webinars, local training)

Access to guideline/protocols/order sets

Access to addictions consult services from ED

Cultural change in department

No licensing restrictions to prescribe buprenorphine

Reduced cost of buprenorphine for patients once out of hospital

More time to have patient in ED for initiation process

${ }^{a}$ Micro-dosing is an induction strategy using an initial small dose that is then gradually titrated up over days to a therapeutic level, which may be a more tolerable strategy with reduced risk of precipitated withdrawal [10]

$E D=$ emergency department, $M D=$ medical doctor, $O U D=$ opioid use disorder, $R N=$ registered nurse, $S W=$ social worker

inductions to utilize treatment protocols such as home inductions or micro-dosing that alleviate need for patient to remain in the ED until in severe withdrawal. Second, there may exist administrative shortcomings in providing the necessary supports for follow-up and allied healthcare provider education to support physician led inductions in the ED.
Despite the current existence of published guidelines, protocols, and other educational resources for the initiation of buprenorphine/naloxone, survey respondents still reported a need for these items. This suggests a need for increased awareness of existing resources, and knowledge translation 
Fig. 1 Emergency physician reported attitudes about starting buprenorphine/naloxone in the ED

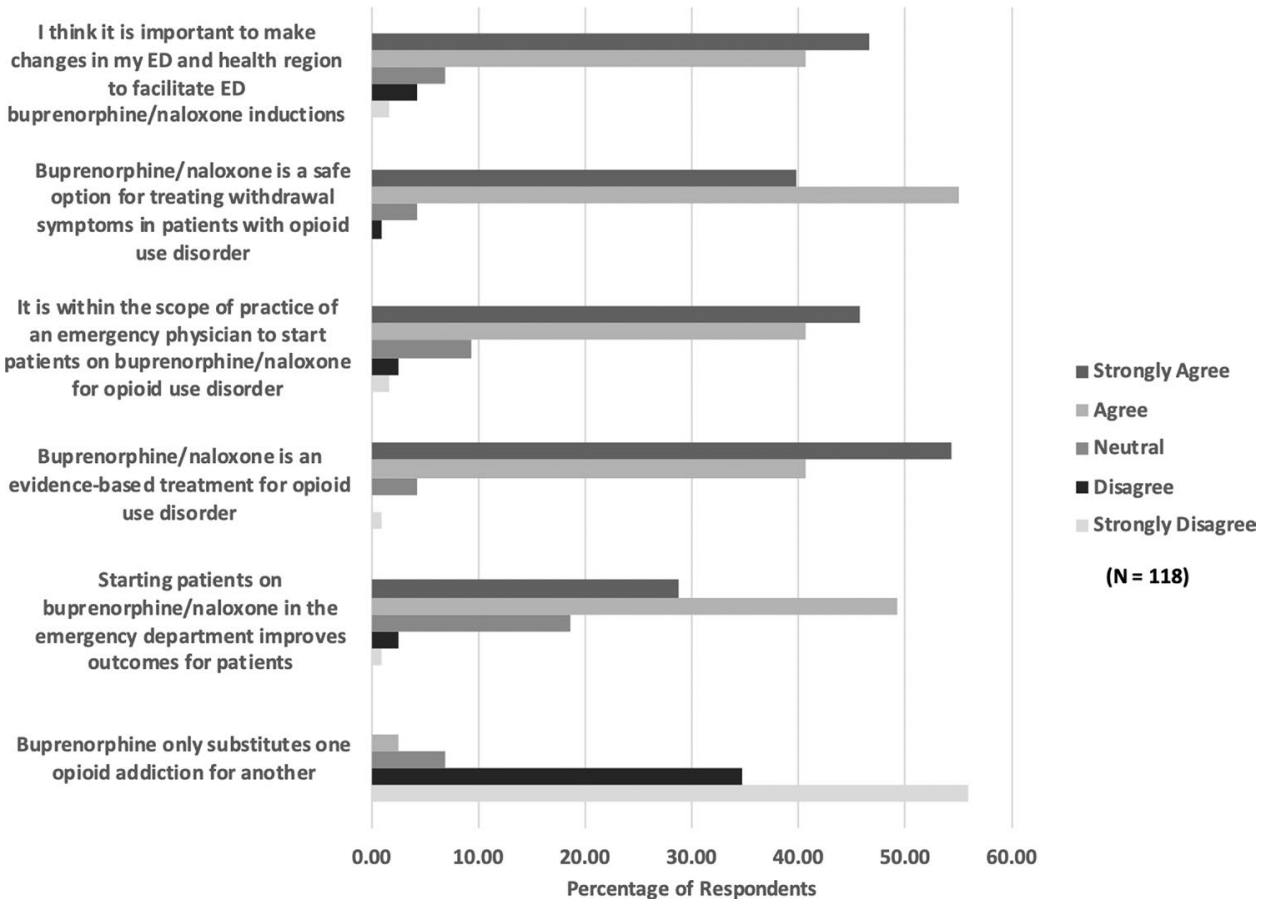

with an administrative focus of local departments to find ways to incorporate and tailor these resources to local needs.

\section{Comparison to previous studies}

The reported barriers in this study were similar to that of US studies with the need for more allied health support, time constraints, and access to timely follow-up being common themes [6-8]. The physician attitudes reported in our study were similar to the findings of the interviews of Canadian emergency physicians performed by Dong et al., that the use of buprenorphine/naloxone in the ED is safe, appropriate, and effective [9]. Our survey findings add further sample size and more representation of physicians practicing in rural practice settings regarding these perceived barriers and attitudes in Canada.

The Dong et al. study identified a key theme of perceived barriers under the title of 'patient onus', which included subthemes such as difficulty engaging patients in care, patient negative perceptions of treatment, and patients unwilling to engage and comply [9]. Our study did not include proposed barriers to capture this theme and rates of these barriers are likely under-represented in our findings. Five (4.2\%) respondents in our study provided optional responses that patient preference was a barrier.

\section{Limitations}

The convenience sampling method and low response rate introduce sampling bias and limit the generalizability of our findings. Physicians who are more supportive of buprenorphine/naloxone use in the ED may have been more likely to access and complete the survey, and the overall positive attitudes are likely overstated. Although limited, we believe the findings still provide a general sense of some of the most frequent barriers and that the overall burden of perceived barriers is high in rural and urban settings.

\section{Conclusion}

In this convenience sampling survey of Canadian emergency physicians, there was a high burden of reported barriers that prevent physicians from initiating patients with opioid use disorder on buprenorphine/naloxone in the ED. The majority of respondents agree that it is important to make changes in their ED better facilitate the practice. Despite the current availability of educational resources, published protocols and guidelines, survey respondents still requested these as a needed resource, suggesting improvement is needed in knowledge translation linking emergency physicians to existing resources and tailoring them to local needs.

Supplementary Information The online version contains supplementary material available at https://doi.org/10.1007/s43678-021-00191-y.

Acknowledgements The authors would like to acknowledge Dr. Patrick McLane and Zainab Suleman from the AHS Emergency Strategic Clinical Network for their advice on things to consider including in our survey based on their work on a multi-site buprenorphine/naloxone quality improvement project. We would also like to acknowledge 
the support of Dr. Catherine Patocka, Dr. Grant Innes, and Dr. James Andruchow who provided support for the conceptualization of this project as part of a University of Calgary Resident Research Course.

\section{References}

1. D'Onofrio G, O'Connor PG, Pantalon MV, Chawarski MC, Busch SH, Owens PH, Bernstein SL, Fiellin DA. Emergency departmentinitiated buprenorphine/naloxone treatment for opioid dependence: a randomized clinical trial. JAMA. 2015;313(16):1636-44. https://doi.org/10.1001/jama.2015.3474.

2. Larochelle MR, Bernson D, Land T, Stopka TJ, Wang N, Xuan Z, Bagley SM, Liebschutz JM, Walley AY. Medication for opioid use disorder after nonfatal opioid overdose and association with mortality: a cohort study. Ann Intern Med. 2018;169(3):137-45. https://doi.org/10.7326/M17-3107.

3. Bruneau J, Ahamad K, Goyer M-È, Poulin G, Selby P, Fischer B, Wild TC, Wood E. Management of opioid use disorders: a national clinical practice guideline. CMAJ. 2018;190(9):E247-57. https://doi.org/10.1503/cmaj.170958.

4. Koh JJ, Klaiman M, Miles I, Cook J, Kumar T, Sheikh H, Dong K, Orkin AM, Ali S, Shouldice E. CAEP Position Statement: Emergency department management of people with opioid use disorder. CJEM. Cambridge University Press; 2020;22(6):768-71.

5. Hoyeck P, Wiercigroch D, Clarke C, Moineddin R, Sheikh H, Hulme J. Understanding current practice of opioid use disorder management in emergency departments across Canada: a crosssectional study. CJEM. 2020;22(4):494-8.

6. Hawk KF, D'Onofrio G, Chawarski MC, O'Connor PG, Cowan E, Lyons MS, Richardson L, Rothman RE, Whiteside LK, Owens PH, Martel SH, Coupet E Jr, Pantalon M, Curry L, Fiellin DA,
Edelman EJ. Barriers and facilitators to clinician readiness to provide emergency department-initiated buprenorphine. JAMA Netw Open. 2020;3(5): e204561. https://doi.org/10.1001/jamanetwor kopen.2020.4561.

7. Im DD, Chary A, Condella AL, Vongsachang H, Carlson LC, Vogel L, Martin A, Kunzler N, Weiner SG, Samuels-Kalow M. Emergency department clinicians' attitudes toward opioid use disorder and emergency department-initiated buprenorphine treatment: a mixed-methods study. West J Emerg Med. 2020;21(2):261-71. https://doi.org/10.5811/westjem.2019.11. 44382.

8. Lowenstein M, Kilaru A, Perrone J, Hemmons J, Abdel-Rahman D, Meisel ZF, Delgado MK. Barriers and facilitators for emergency department initiation of buprenorphine: A physician survey. Am J Emerg Med. 2019;37(9):1787-90. https://doi.org/10.1016/j. ajem.2019.02.025.

9. Dong KA, Lavergne KJ, Salvalaggio G, Weber SM, Xue CJ, Kestler A, Kaczorowski J, Orkin AM, Pugh A, Hyshka E. Emergency physician perspectives on initiating buprenorphine/naloxone in the emergency department: A qualitative study. JACEP Open. 2021;2: e12409. https://doi.org/10.1002/emp2.12409.

10. Randhawa PA, Brar R, Nolan S. Buprenorphine-naloxone "microdosing": an alternative induction approach for the treatment of opioid use disorder in the wake of North America's increasingly potent illicit drug market. Can Med Assoc J. 2020;192(3), E73 LP-E73. https://doi.org/10.1503/cmaj.74018 